\title{
Gibbs Sampler Based Control of Autonomous Vehicle Swarms in the Presence of Sensor Errors*
}

\author{
Wei Xi and John S. Baras
}

\begin{abstract}
In earlier work of the authors[1], [2], [3], [4], [5], it was shown that Gibbs sampler based annealing algorithms could be used for vehicle swarms to achieve self-organization. Nevertheless, the earlier convergence analyses were based on the assumption that the Gibbs potential can be precisely evaluated. In practice, Gibbs potentials have to be measured via sensors, which usually introduce errors. The robustness of the stochastic algorithm under sensor errors is studied in this paper. Two types of sensor error, range-error and randomerror, are investigated. Analytical results on convergence are derived for sensor errors with limited support, and are further validated through simulations.
\end{abstract}

\section{INTRODUCTION}

In recent years, with the rapid advances in sensing, communication, computation, and actuation capabilities, groups (or swarms) of autonomous unmanned vehicles (AUVs) are expected to cooperatively perform dangerous or explorative tasks in a broad range of potential applications [6]. Due to the large scale of vehicle networks and bandwidth constraints on communication, distributed methods for control and coordination of these autonomous swarms are especially appealing [7], [8], [9], [10], [11].

A popular distributed approach is based on artificial potential functions (APF), which encode desired vehicle behaviors such as inter-vehicle interactions, obstacle avoidance, and target approaching [12], [13], [14], [15]. Despite its simple, local, and elegant nature, this approach suffers from the problem that the system dynamics could be trapped at the local minima of potential functions [16]. Researchers attempted to address this problem by designing potential functions that have no other local minima [17], [18], or escaping from local minima using ad hoc techniques, e.g., random walk [19], virtual obstacles [20], and virtual local targets [21].

An alternative approach to dealing with the above problem was explored using the concept of Markov Random Fields (MRFs) by Baras and Tan [1]. Traditionally used in statistical mechanics and in image processing [22], MRFs were proposed to model swarms of vehicles. Similar to the APF approach, global objectives and constraints (e.g., obstacles) are reflected through the design of potential functions. The movement of vehicles is then decided using simulated

\footnotetext{
*This research was supported by the Army Research Office under the ODDR\&E MURI01 Program Grant No. DAAD19-01-1-0465 to the Center for Networked Communicating Control Systems (through Boston University), and under ARO Grant No. DAAD190210319.

$\mathrm{W}$. Xi and J. S. Baras are with the Institute for Systems Research and the Department of Electrical \& Computer Engineering, University of Maryland, College Park, MD 20742, USA. $\{$ wxi, baras $\}$ @isr.umd.edu
}

annealing based on the Gibbs sampler. Theoretical studies have shown that, with this approach, it is possible to achieve desired configurations with minimum potential despite the presence of local minima, which was further confirmed by simulations [2], [3]. Due to the stochastic nature and sequential location updating, slow convergence rate prevents the stochastic algorithm to be used in practice. To deal with these problems, parallel sampling techniques and a hybrid scheme were proposed to reduce the traveling time [4], [1].

However, an underlying assumption in our previous studies was that the potential function can be precisely evaluated. In practice, the potential values are calculated using sensor measurements. In many applications, e.g., battle field scenario, cost-effective sensors are preferred to reduce the total expense. As a result, sensor uncertainties introduce noise to Gibbs potential evaluations. It is then of interests to study the robustness of the annealing algorithm. In the past, this issue has been studied for the annealing algorithm based on classical MRF. Grover presented an early analysis of the impact of fixed range-error on equilibrium properties [23]. Gelfand and Mitter studied the effects of state-independent Gaussian noise. They showed that in certain conditions, slowly decreasing random-error will not affect the limiting configurations [24], [25]. Greening studied the impact of errors for the Metropolis annealing algorithm [26]. In this paper, we investigate the impact of both fixed range-error and bounded random-error on the annealing algorithm proposed in [3]. In our analysis, unlike previous studies, we do not assume that the randomerror follows a Gaussian distribution. Sufficient conditions that guarantee the convergence to the global minimizer are derived. Simulations confirm the analysis results.

The remainder of the paper is organized as follows. In section II, a battle field scenario is described, which was used for the illustration of the problem formulation. Then, the Gibbs sampler based algorithm and the convergence analysis are revisited for the convenience of the readers. Section III, investigates the convergence and equilibrium properties of the annealing algorithm with inaccurate Gibbs potential. Simulation results and conclusions are provided in sections IV and V.

\section{REVIEW OF GIBBS SAMPLER BASED ALGORITHM}

\section{A. MRFs and Gibbs Sampler}

One can refer to, e.g., [22], [27], for a review of MRFs. Let $S$ be a finite set of cardinality $\sigma$, with elements indexed by $s$ and called sites. For $s \in S$, let $\Lambda_{s}$ be a finite set called the phase space for site $s$. A random field on $S$ 
is a collection $X=\left\{X_{s}\right\}_{s \in S}$ of random variables $X_{s}$ taking values in $\Lambda_{s}$. A configuration of the system is described by $x=\left\{x_{s}, s \in S\right\}$, where $x_{s} \in \Lambda_{s}, \forall s$. The product space $\Lambda_{1} \times \cdots \times \Lambda_{\sigma}$ is called the configuration space. A neighborhood system on $S$ is a family $\mathcal{N}=$ $\left\{\mathcal{N}_{s}\right\}_{s \in S}$, where $\forall s, r \in S, \mathcal{N}_{s} \subset S, s \notin \mathcal{N}_{s}$, and $r \in \mathcal{N}_{s}$ if and only if $s \in \mathcal{N}_{r}$.

$\mathcal{N}_{s}$ is called the neighborhood of site $s$. The random field $X$ is called a Markov random field (MRF) with respect to the neighborhood system $\mathcal{N}$ if, $\forall s \in S, P\left(X_{s} \mid X_{S \backslash s}\right)=$ $P\left(X_{s} \mid X_{r}, r \in \mathcal{N}_{s}\right)$.

A random field $X$ is a Gibbs random field if and only if it has the Gibbs distribution:

$$
P(X=x)=\frac{e^{-\frac{U(x)}{T}}}{Z}, \forall x,
$$

where $T$ is the temperature variable (widely used in simulated annealing algorithms), $U(x)$ is the potential (or energy) of the configuration $x$, and $Z$ is the normalizing constant, called the partition function: $Z=\sum_{x} e^{-\frac{U(x)}{T}}$. One then considers the following useful class of potential functions $U(x)=\sum_{s \in \Lambda} \Phi_{s}(x)$, which is a sum of individual contributions $\Phi_{s}$ evaluated at each site. The Hammersley-Clifford theorem [27] establishes the equivalence of a Gibbs random field and an MRF.

The Gibbs sampler belongs to the class of Markov Chain Monte Carlo (MCMC) methods, which sample Markov chains leading to stationary distributions. The algorithm updates the configuration by visiting sites sequentially or randomly with certain proposal distribution [22], and sampling from the local specifications of a Gibbs field. A sweep refers to one round of sequential visits to all sites, or $\sigma$ random visits under the proposal distribution. The convergence of the Gibbs sampler was studied by D. Geman and S. Geman in the context of image processing [28]. There it was shown that as the number of sweeps goes to infinity, the distribution of $X(n)$ converges to the Gibbs distribution $\Pi$. Furthermore, with an appropriate cooling schedule, simulated annealing using the Gibbs sampler yields a uniform distribution on the set of minimizers of $U(x)$. Thus the global objectives could be achieved through appropriate design of the Gibbs potential function.

\section{B. Problem Setup}

Consider a 2D mission space (the extension to 3D space is straightforward), which is discretized into a lattice of cells. For ease of presentation, each cell is assumed to be square with unit dimensions. One could of course define cells of other geometries (e.g., hexagons) and of other dimensions (related to the coarseness of the grid) depending on the problems at hand. Label each cell with its coordinates $(i, j)$, where $1 \leq i \leq N_{1}, 1 \leq j \leq N_{2}$, for $N_{1}, N_{2}>0$. There is a set of vehicles (or mobile nodes) $S$ indexed by $s=1, \cdots, \sigma$ on the mission space. To be precise, each vehicle $s$ is assumed to be a point mass located at the center of some cell $\left(i_{s}, j_{s}\right)$, and the position of vehicle $s$ is taken to be $p_{s}=\left(i_{s}, j_{s}\right)$. At most one vehicle is allowed to stay in each cell at any time instant.

The distance between two cells, $\left(i_{a}, j_{a}\right)$ and $\left(i_{b}, j_{b}\right)$, is defined to be

$$
R \triangleq\left\|\left(i_{a}, j_{a}\right)-\left(i_{b}, j_{b}\right)\right\|=\sqrt{\left(i_{a}-i_{b}\right)^{2}+\left(j_{a}-j_{b}\right)^{2}} .
$$

There might be multiple obstacles in the space, where an obstacle is defined to be a set of adjacent cells that are inaccessible to vehicles. For instance, a "circular" obstacle centered at $p^{o k}=\left(i^{o k}, j^{o k}\right)$ with radius $R^{o k}$ can be defined as $O \triangleq\left\{(i, j): \sqrt{\left(i-i^{o k}\right)^{2}+\left(j-j^{o k}\right)^{2}} \leq R^{o}\right\}$. There can be at most one target area in the space. A target area is a set of adjacent cells that represent desirable destinations of mobile nodes. A "circular" target area with its center at $p^{g}$ can be defined similarly as a "circular" obstacle. An example mission scenario is shown in Fig. 1.

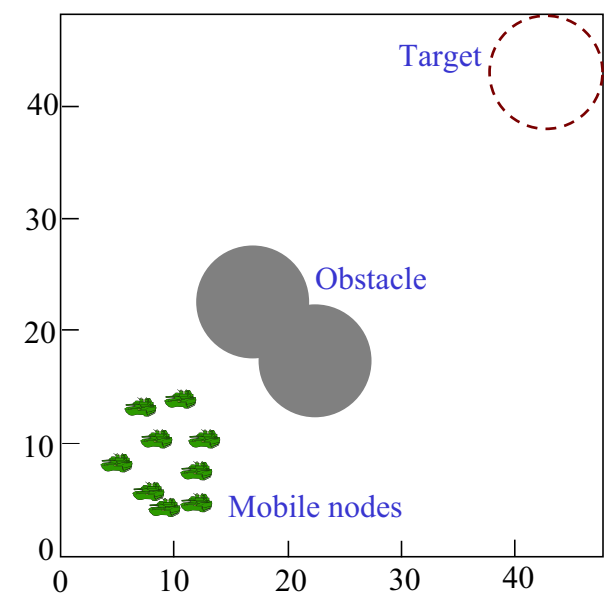

Fig. 1. An example mission scenario with a circular target and a nonconvex obstacle (formed by two overlapping circular obstacles). Note that since the mission space is a discretized grid, a cell is taken to be within a disk if its center is so.

In this paper all vehicles are assumed to be identical. Each vehicle has a sensing range $R_{s}$ : it can detect whether a cell within distance $R_{s}$ is occupied by some node or obstacle through sensing or direct inter-vehicle communication. The moving decision of each node $s$ depends on other nodes located within distance $R_{i}\left(R_{i} \leq R_{s}\right)$, called the interaction range. These nodes form the set $\mathcal{N}_{s}$ of neighbors of node $s$. A node can travel at most $R_{m}\left(R_{m} \leq R_{s}\right)$, called moving range, within one move. See Fig. 2 for illustration of these range definitions.

The neighborhood system defined earlier naturally leads to a dynamic graph, where each vehicle represents a vertex of the graph and the neighborhood relation prescribes the edges between vehicles. An MRF can then be defined on the graph, where each vehicle $s$ is a site and the associated phase space $\Lambda_{s}$ is the set of all cells located within the moving range $R_{m}$ from location $p_{s}$ and not occupied by obstacles or other vehicles. The configuration space of the MRF is denoted as $\mathcal{X}$. 


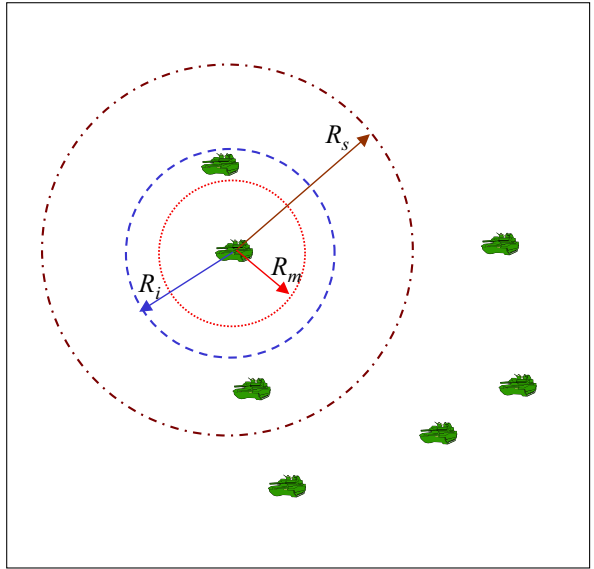

Fig. 2. Illustration of the sensing range $R_{s}$, the interaction range $R_{i}$, and the moving range $R_{m}$.

The Gibbs potential $U(x)=\sum_{s} \Phi_{s}(x)$, where $\Phi_{s}(x)$ is considered to be a summation of all clique potentials $\Psi_{c}(x)$, and depends only on $x_{s}$ and $\left\{x_{r}, r \in \mathcal{N}_{s}\right\}$. The clique potentials $\Psi_{c}(x)$ are used to describe local interactions depending on applications. Specifically,

$$
\Phi_{s}(x)=\sum_{c \ni s} \Psi_{c}=\Psi_{\{s\}}\left(x_{s}\right)+\sum_{r \in \mathcal{N}_{s}} \Psi_{\{s, r\}}\left(x_{s}, x_{r}\right) .
$$

\section{Gibbs sampler based algorithm}

In [3], a two-step annealing algorithm was proposed to coordinate maneuvering autonomous swarms to achieve a global task. In this subsection, we briefly review the algorithm and some convergence analysis results.

Before stating the algorithm, we first introduce a key idea involved, which is the configuration-and-temperaturedependent proposal distribution $G_{T}^{x}(s)$. In particular, given a configuration $x$ and a temperature $\underset{U(z)}{T}$

$$
G_{T}^{x}(s)=\frac{\sum_{z \in \mathcal{N}_{m}^{x}(s)} e^{-\frac{U(\hat{z})}{T}}}{\sum_{s^{\prime} \in S} \sum_{z \in \mathcal{N}_{m}^{x}\left(s^{\prime}\right)} e^{-\frac{U(z)}{T}} .}
$$

In (2) $\mathcal{N}_{m}^{x}(s)$ denotes the set of s-neighbors of configuration $x$ within one move:

$$
\mathcal{N}_{m}^{x}(s) \triangleq\left\{z: z_{S \backslash s}=x_{S \backslash s},\left\|z_{s}-x_{s}\right\| \leq R_{m}\right\},
$$

where $S \backslash s$ denotes the set of all nodes except $s$.

Let $D_{T}^{x}(s)=\sum_{z \in \mathcal{N}_{m}^{x}(s)} e^{-\frac{\Phi_{s}(z)-\Phi_{s}(x)}{T}}$. It could be also easily verified that

$$
G_{T}^{x}(s)=\frac{D_{T}^{x}(s)}{\sum_{s^{\prime}} D_{T}^{x}\left(s^{\prime}\right)} .
$$

Note that each node $s$ would be able to evaluate $D_{T}^{x}(s)$ locally if $R_{s} \geq R_{i}+R_{m}$.

Picking an appropriate cooling schedule $T(n)$ and a sufficiently large $N_{\max }$, the algorithm briefly works as follows.

Initially, all nodes evaluate and send $D_{T(1)}^{x(0)}(s)$ to a preselected node $s(0)$. Node $s(0)$ then calculates and samples the proposal distribution according to (3) in order to select the next node $s^{1}(1)$ for updating. Node $s(0)$ notifies $s^{1}(1)$ and sends the vector $\left\{D_{T(1)}^{x(0)}(s), s \in S\right\}$. Node $s^{1}(1)$ updates its location by locally sampling possible vacant cells $l$ using Gibbs sampler:

$$
P\left(x_{s}=l\right)=\frac{e^{-\frac{\Phi_{s}\left(x_{s}=l, x_{S \backslash s}\right)}{T}}}{\sum_{l^{\prime} \in C_{m}^{s}} e^{-\frac{\Phi_{s}\left(x_{s}=l^{\prime}, x_{S \backslash s}\right)}{T}}} .
$$

Node $s^{1}(1)$ then asks its neighbors to recalculate and resend $D_{T(1)}^{x(1)}(s)$ to update the vector $\left\{D_{T(1)}^{x(0)}(s), s \in S\right\}$. Node $s^{1}(1)$ reevaluates the proposal distribution and samples it to select the next node for updating. The process is repeated until the temperature is reduced to $T\left(N_{\max }\right)$.

\section{Convergence analysis results}

Let $P_{T}$ denote the Markov kernel defined by the random update scheme (2) and (4), i.e.,

$$
\begin{aligned}
& P_{T}(x, y) \triangleq \operatorname{Pr}(X(n+1)=y \mid X(n)=x) \\
& =\sum_{s \in S} \frac{e^{-\frac{U(y)}{T}} \cdot \mathbf{1}\left(y \in \mathcal{N}_{m}^{x}(s)\right)}{\sum_{s^{\prime} \in S} \sum_{z \in \mathcal{N}_{m}^{x}\left(s^{\prime}\right)} e^{-\frac{U(z)}{T}}} .
\end{aligned}
$$

For a fixed temperature, the equilibrium distribution can be expressed in the following theorem.

Theorem 2.1: The Markov kernel $P_{T}$ has a unique stationary distribution $\Pi_{T}$ with

$$
\Pi_{T}(x)=\frac{e^{-\frac{U(x)}{T}} \sum_{s \in S} \sum_{z \in \mathcal{N}_{m}^{x}(s)} e^{-\frac{U(z)}{T}}}{Z_{T}},
$$

where $Z_{T}=\sum_{y} e^{-\frac{U(y)}{T}} \sum_{s \in S} \sum_{z \in \mathcal{N}_{m}^{y}(s)} e^{-\frac{U(z)}{T}}$ is the partition function.

Theorem 2.2: Let $\{T(n)\}$ be a cooling schedule decreasing to 0 such that eventually, $T(n) \geq \frac{\tau \Delta}{\ln n}$, where $\tau$ is the minimum number of steps to ensure the Markov chain kernel $P_{T}$ has a strictly positive power and $\Delta=$ $\max _{x, y}\left\{\|U(x)-U(y)\|_{1}:\left\|p_{x}-p_{y}\right\| \leq R_{m}\right\}$. Let $\mathcal{M}$ be the set of global minima of $U(\cdot)$. Then for any initial distribution $\nu$,

$$
\lim _{n \rightarrow \infty} \nu P_{1} \cdots P_{n} \rightarrow \Pi_{\infty}
$$

where $\Pi_{\infty}$ is the distribution (6) evaluated at $T=0$. In particular,

$$
\sum_{x \in \mathcal{M}} \Pi_{\infty}(x)=1
$$

One can refer to [3] for the detailed proofs.

\section{Convergence Analysis with Gibbs potential INACCURACY}

In this section, we study the impact of sensor errors on the convergence properties of the annealing algorithm described in subsection II-C. The sensor errors considered in this paper fall into two categories: range-error and randomerror. A potential function is said to have range-errors if the difference between the nominal potential value $U(x)$ 
and the observed one $\hat{U}(x)$ is confined to a fixed range, and does not change with time. The range-error is usually caused by the systematic error of defective sensors. On the contrary, we consider that the potential function $\tilde{U}(x)$ has random-errors if the difference between $U(x)$ and $\tilde{U}(x)$ is an independent random variable, which is denoted as $Z(x)$. The random-error introduces time-varying potential evaluations. In what follows, the convergence properties under these two types of sensor errors will be analyzed respectively.

\section{A. Gibbs potential with range-error}

When sensors carried by vehicles have range-error, the observed potential $\hat{U}(x)$ of a configuration $x$ can be expressed as

$$
\hat{U}(x)=U(x)+e(x),
$$

where $e(x)$ is a finite constant. We assume $\underline{e} \leq e(x) \leq \bar{e}$, where $\underline{e}$ and $\bar{e}$ are the upper bound and lower bound of range error respectively. The observed potential $\hat{U}(x)$ satisfies

$$
U(x)+\underline{e} \leq \hat{U}(x) \leq U(x)+\bar{e},
$$

Since the range-error is time-invariant, the Gibbs sampler defines a homogeneous Markov chain at a fixed temperature $T$. By directly applying Theorem 2.1, one could conclude that there exist a unique equilibrium distribution $\hat{\pi}_{T}$ at temperature $T$,

$$
\hat{\Pi}_{T}(x)=\frac{e^{-\frac{\hat{U}(x)}{T}} \sum_{s \in S} \sum_{z \in \mathcal{N}_{m}^{x}(s)} e^{-\frac{\hat{U}(z)}{T}}}{Z_{T}} .
$$

Proposition 3.1: Let $\Pi_{T}(x)$ denote the equilibrium distribution in (6). Let the maximum oscillation of range errors be $\Delta_{e}=\bar{e}-\underline{e}$. Then,

$$
e^{-\frac{2 \Delta_{e}}{T}} \Pi_{T}(x) \leq \hat{\Pi}_{T}(x) \leq e^{\frac{2 \Delta_{e}}{T}} \Pi_{T}(x)
$$

Moreover

$$
\left\|\hat{\Pi}_{T}-\Pi_{T}\right\| \leq e^{\frac{2 \Delta_{e}}{T}}-1,
$$

where $\|\cdot\|$ stands for $L_{1}$ norm.

Proof. Pick any configuration $x \in \mathcal{X}$. For each configuration $y \in\left\{x \cup \mathcal{N}^{m}(x)\right\}$, let $U(y)=U(y)+\underline{e}$. For any other configuration $\left(x^{\prime} \in\left\{x \cup \mathcal{N}^{m}(x)\right\}^{c}\right)$, let $\hat{U}\left(x^{\prime}\right)=U\left(x^{\prime}\right)+\bar{e}$. Then, we have

$$
\hat{\Pi}_{T}(x) \leq \frac{e^{-\frac{U(x)+e}{T}} \sum_{s \in S} \sum_{y \in \mathcal{N}^{m}(x)} e^{-\frac{U(y)+e}{T}}}{Z_{T}(\hat{U})},
$$

where $Z_{T}(\hat{U})$ denotes the partition function for the Gibbs potential $\hat{U}(x)$. Clearly,

$$
Z_{T}(\hat{U})>Z_{T}(U+\bar{e}) .
$$

Then,

$$
\begin{aligned}
\hat{\Pi}_{T}(x) & \leq \frac{e^{-\frac{2 e}{T}} e^{-\frac{U(x)}{T}} \sum_{s \in S} \sum_{y \in \mathcal{N}^{m}(x)} e^{-\frac{U(y)}{T}}}{Z_{T}(U+\bar{e})} \\
& =\frac{e^{-\frac{2 e}{T}} e^{-\frac{U(x)}{T}} \sum_{s \in S} \sum_{y \in \mathcal{N}^{m}(x)} e^{-\frac{U(y)}{T}}}{e^{-\frac{2 \bar{e}}{T}} Z_{T}(U)} \\
& =e^{\frac{2(\bar{e}-e)}{T}} \pi_{T}(x)=e^{\frac{2 \Delta_{e}}{T}} \pi_{T}(x) .
\end{aligned}
$$

The converse argument supplies the lower bound. Then, By inequality (12)

$$
\begin{aligned}
\left\|\hat{\Pi}_{T}-\Pi_{T}\right\| \leq & \max \left\{\left\|e^{\frac{2 \Delta_{e}}{T}} \Pi_{T}-\Pi_{T}\right\|,\right. \\
& \left.\left\|e^{-\frac{2 \Delta \Delta_{e}}{T}} \Pi_{T}-\Pi_{T}\right\|\right\}=e^{\frac{2 \Delta_{e}}{T}}-1 .
\end{aligned}
$$

The last equality holds because $e^{\frac{2 \Delta_{e}}{T}}-1>1-e^{-\frac{2 \Delta_{e}}{T}}$.

Proposition 3.1 unveils the basic impact of range-error on the equilibrium distribution for a fixed temperature $T$. Moreover, by picking an appropriate cooling schedule as in Theorem 2.2, i.e., logarithmic cooling rate, it can be shown that the SA algorithm leads to limiting configurations with minimum energy $\hat{U}(x)$. If the global minimizer of $\hat{U}(x)$ minimizes the nominal Gibbs potential $U(x)$, the rangeerror does not affect limiting configurations. A sufficient condition is formally stated in the following proposition.

Proposition 3.2: For the Gibbs potential with rangeerror, the simulated annealing algorithm leads to the global minimizer $x^{*}$ of the nominal Gibbs potential $U(x)$, if the following condition is satisfied:

$$
\Delta_{e} \leq \frac{1}{2} \Delta_{U}
$$

where $\Delta_{U}$ is the minimum potential difference with global minimizer, i.e.,

$$
\Delta_{U}=\min _{x \in \mathcal{X}, x \neq x^{*}}\left|U(x)-U\left(x^{*}\right)\right| .
$$

Proof. Let $x$ be any configuration other than the global minimizer $x^{*}$, i.e., $x \neq x^{*} \in \mathcal{X}$. By equation (10), we have

$$
\begin{aligned}
\hat{U}\left(x^{*}\right) & \leq U\left(x^{*}\right)+\Delta_{e} \leq U(x)-\Delta_{U}+\Delta_{e} \\
& \leq U(x)-\Delta_{e} \leq \hat{U}(x) .
\end{aligned}
$$

One then concludes that $x^{*}$ minimizes the potential function $\hat{U}(x) \square$

If the maximum oscillation of range-error is too large, the simulated annealing algorithm may not be able to lead the limiting configurations to the global minimizer.

\section{B. Gibbs potential with random-error}

In the previous section, the potential error $e(x)$ is assumed to be a fixed value for each configuration $x$. In practice, the potential error due to sensor noise usually varies with time, i.e., the Gibbs potential has random-errors. For ease of analysis, let the random-error $Z_{x}$ be an independent random variable associated with each configuration $x$. The observed Gibbs potential $\tilde{U}$ with random-error can then be expressed as

$$
\tilde{U}\left(x, z_{x}\right)=U(x)+Z_{x},
$$

where $Z_{x}$ follows a probability distribution $f_{z_{x}}$

Proposition 3.3: Let $Z=\left\{Z_{x}: x \in \mathcal{X}\right\}$ be the vector of random-errors. The Gibbs sampler with random-errors defines a homogenous Markov chain at a fixed temperature with kernel matrix satisfying

$$
\tilde{P}_{T}=E_{Z}\left(P_{T}(z)\right) .
$$


where $P_{T}(z)$ is the kernel matrix with fixed range-error $z$. Moreover, there exist a unique equilibrium distribution $\tilde{\Pi}_{T}$ at a fixed temperature $T$. Starting from any initial distribution $\nu_{T}^{0}$,

$$
\lim _{n \rightarrow \infty}\left\|\nu_{T}^{0}\left(\tilde{P}_{T}\right)^{n}-\tilde{\Pi}_{T}\right\|=0
$$

Proof. For any two configurations $x, y \in \mathcal{X}$, the transition probability $\tilde{p}(x, y)$ satisfies

$$
\tilde{p}_{T}(x, y)=\int p_{T}(x, y \mid Z=z) f(z) d z .
$$

One could then conclude that (16) holds. Given any fixed range-error $z$, we know that the kernel matrix $P_{T}(z)$ is primitive, i.e., the Markov chain is irreducible and aperiodic. Since $\tilde{P}_{T}$ is a superposition of $P_{T}(z)$, the primitivity of $\tilde{P}_{T}$ is obvious. The uniqueness and existence of the equilibrium distribution then follow accordingly. The final statement follows from the ergodicity of the primitive Markov chain.

Unfortunately, the lack of an explicit form of the stationary distribution for the Markov chain $\tilde{P}_{T}$ presents challenges for analyzing the robustness of the SA algorithm under random-errors. To simplify the analysis, we assume that the random-error has only limited support. Similar ideas as those used for analyzing range-errors in the previous subsection can then be applied.

Proposition 3.4: Assume that the random-error $z$ is bounded, i.e., $\underline{z} \leq z_{x} \leq \bar{z}, \forall x$. Let $\Delta_{z}=\bar{z}-\underline{z}$. Let $C\left(P_{T}\right)$ be the contraction coefficient of a Markov kernel $P_{T}$ (see [22]). The equilibrium distribution $\tilde{\Pi}_{T}$ satisfies the following inequality:

$$
\left\|\tilde{\Pi}_{T}-\Pi_{T}\right\| \leq \frac{e^{\frac{2 \Delta_{z}}{T}}-1}{1-C\left(\tilde{P}_{T}\right)}
$$

Proof. By assumption, since $Z$ is bounded, given any $z \in$ $Z$, it is easy to show that, for all $x, y \in \mathcal{X}$, the matrix $P_{T}(Z=z)$ satisfies

$$
e^{-\frac{2 \Delta z}{T}} P_{T}(x, y) \leq P_{T}(x, y \mid Z=z) \leq e^{\frac{2 \Delta z}{T}} P_{T}(x, y),
$$

where $P_{T}=P_{T}(Z=0)$ is the Markov chain kernel matrix with nominal Gibbs potential. Then

$$
\begin{aligned}
\left\|\tilde{\Pi}_{T}-\Pi_{T}\right\| & =\| \tilde{\Pi}_{T} \tilde{P}_{T}-\Pi_{T} \tilde{P}_{T}+\Pi_{T} \tilde{P}_{T}-\Pi_{T} P_{T} \mid \\
& \leq\left\|\tilde{\Pi}_{T}-\Pi_{T}\right\| C\left(\tilde{P}_{T}\right)+\| \Pi_{T} \tilde{P}_{T}-\Pi_{T} P_{T} \mid \\
& \leq\left\|\tilde{\Pi}_{T}-\Pi_{T}\right\| C\left(\tilde{P}_{T}\right)+\left(e^{\frac{2 \Delta_{z}}{T}}-1\right) .
\end{aligned}
$$

This is equivalent to

$$
\left\|\tilde{\Pi}_{T}-\Pi_{T}\right\|\left(1-C\left(\tilde{P}_{T}\right)\right) \leq e^{\frac{2 \Delta_{z}}{T}}-1 .
$$

The inequality (19) then follows.

Clearly, as the maximum oscillation of the random-errors $\Delta_{z}$ tends to zero, the distribution $\nu_{T}^{n}=\nu_{T}^{0}\left(\tilde{P}_{T}\right)^{n}$ tends to approach the nominal equilibrium distribution $\Pi_{T}$.

Proposition 3.5: Pick an appropriate cooling schedule $T(n)$ such that $\lim _{n \rightarrow \infty} T(n)=0$ and the Markov chain
$\tilde{P}_{T}$ converges as temperature tends to zero. Assume $\Delta_{z} \leq$ $\frac{1}{2} \Delta_{U}$. Then, From any initial distribution $\nu$

$$
\lim _{n \rightarrow \infty} \nu \prod_{i=1}^{n} \tilde{P}_{T(i)}=\Pi_{\infty},
$$

i.e., the limiting configurations tends to global minimizers of the nominal potential $U(x)$.

Proof. Since the Markov chain kernel matrix $\left\{\tilde{P}_{T(i)}\right\}$ is primitive, by picking an appropriate logarithmic cooling schedule $(e . g ., T(n)=c / \log (n))$, the simulated annealing algorithm converges to a limiting distribution $\tilde{\Pi}_{\infty}$, i.e., $\lim _{n \rightarrow \infty} \nu \prod_{i=1}^{n} \tilde{P}_{T(i)}=\tilde{\Pi}_{\infty}$, where $\tilde{\Pi}_{\infty}=\lim _{T \rightarrow 0} \tilde{\Pi}_{T}$. Next, we will show that the limiting distribution $\tilde{\Pi}_{\infty}$ actually equals $\Pi_{\infty}$.

Let $\Pi_{T}(z)$ denote the equilibrium distribution of the Markov chain kernel $P_{T}(z)$. For any $w \in Z$, one has

$$
\begin{aligned}
& \Pi_{T}(w) \tilde{P}_{T}=\Pi_{T}(w) \int_{z} P_{T}(z) f(z) d z \\
= & \int_{z}\left(\Pi_{T}(w)-\Pi_{T}(z)\right) P_{T}(z) f(z) d z+\int_{z} \Pi_{T}(z) f(z) d z .
\end{aligned}
$$

Let $\bar{\Pi}$ be the mean of $\Pi(z)$ with respect to the probability distribution $f(z), \bar{\Pi}=\int_{z} \Pi_{T}(z) f(z) d z$. Let $\Delta_{\Pi_{T}}=$ $\int_{w} \int_{z}\left(\Pi_{T}(w)-\Pi_{T}(z)\right)\left(P_{T}(z)-P_{T}(w)\right) f(z) d z$. Integrating both sides of (22) with respect to $w$, one then has

$$
\begin{aligned}
& \bar{\Pi}_{T} \tilde{P}=\int_{w} \Pi_{T}(w) \tilde{P}_{T} d w \\
= & \int_{w} f(w) \int_{z} P_{T}(z)\left(\Pi_{T}(w)-\Pi_{T}(z)\right) f(z) d z d w \\
+ & \int_{w} f(w) \int_{z} \Pi_{T}(z) f(z) d z d w \\
= & \frac{1}{2}\left\{\int_{w} \int_{z}\left(\Pi_{T}(w)-\Pi_{T}(z)\right) P_{T}(z) f(z) f(w) d z d w\right. \\
- & \left.\int_{w} \int_{z}\left(\Pi_{T}(w)-\Pi_{T}(z)\right) P_{T}(w) f(z) f(w) d z d w\right\} \\
+ & \int_{w} f(w) \bar{\Pi}_{T} d w \\
= & \frac{1}{2} \Delta_{\Pi_{T}}+\bar{\Pi}_{T}
\end{aligned}
$$

The primitivity of $\tilde{P}$ implies that $\lim _{n \rightarrow \infty} \bar{\Pi}_{T} \tilde{P}^{n}=\tilde{\Pi}_{T}$. Assuming $\left(I-\tilde{P}_{T}\right)^{-1}$ exists, with (22), the left hand side of the above equation can be rewritten as

$$
\begin{aligned}
\bar{\Pi}_{T} \tilde{P}^{n} & =\sum_{i=1}^{n} \frac{1}{2} \Delta_{\Pi_{T}} \tilde{P}_{T}^{i-1}+\bar{\Pi}_{T} \\
& =\frac{1}{2} \Delta_{\Pi_{T}}\left(I-\tilde{P}_{T}^{n}\right)\left(I-\tilde{P}_{T}\right)^{-1}+\bar{\Pi}_{T}
\end{aligned}
$$

As $n$ tends to $\infty$, the equilibrium distribution can then be explicitly expressed as

$$
\tilde{\Pi}_{T}=\frac{1}{2} \Delta_{\Pi_{T}}\left(I-\tilde{P}_{T}^{\infty}\right)\left(I-\tilde{P}_{T}\right)^{-1}+\bar{\Pi}_{T}
$$

By proposition 3.2, one has $\lim _{T \rightarrow 0} \Pi_{T}(z)=\Pi_{\infty}, \forall z$, since $\Delta_{z} \leq \frac{1}{2} \Delta_{U}$. Then, we have

$$
\lim _{T \rightarrow 0} \Delta_{\Pi_{T}}=0, \text { and } \lim _{T \rightarrow 0} \bar{\Pi}_{T}=\Pi_{\infty} .
$$


Take the limit of $T$ for equation (23), and plug in the above equations. The final conclusion then follows:

$$
\begin{aligned}
\tilde{\Pi}_{\infty} & =\lim _{T \rightarrow 0}\left(\frac{1}{2} \Delta_{\Pi_{T}}\left(I-\tilde{P}_{T}^{\infty}\right)\left(I-\tilde{P}_{T}\right)^{-1}+\bar{\Pi}_{T}\right) \\
& =\Pi_{\infty} \cdot \square
\end{aligned}
$$

The result shows that if the bound of the random-error is constrained by $\frac{\Delta_{U}}{2}$, an appropriate cooling schedule leads to global minimizers.

\section{Simulation Results}

Simulations were conducted to verify the robustness analysis of the previous section. A formation control example involving inter-vehicle interactions was used to demonstrate the impact of the sensor error on the convergence of the Gibbs sampler based approach. Other objectives or constraints, such as target-approaching and obstacle avoidance, can be similarly analyzed.

The goal of the simulations is to have the nodes to form (square) lattice structures with a desired inter-vehicle distance $R_{\text {des }}$. The potential function used was:

$$
U(x)=\sum_{r \neq s,\left\|x_{r}-x_{s}\right\| \leq R_{i}} c_{1}\left(\left|\left\|x_{r}-x_{s}\right\|-R_{d e s}\right|^{\alpha}-c_{2}\right),
$$

where $c_{1}>0, c_{2}>0$, and $\alpha>0$. A proper choice of $c_{2}$ encourages nodes to have more neighbors. The power $\alpha$ shapes the potential function. In particular, for $\left\|\mid x_{r}-x_{s}\right\|-$ $R_{\text {des }} \mid<1$, smaller $\alpha$ leads to larger potential difference from the global minimum.

In these simulations, 9 nodes were initially randomly placed on an 8 by 8 grid (see Fig. 3 (a)). Parameters used were: $R_{i}=4 \sqrt{2}-\epsilon, R_{m}=2 \sqrt{2}+\epsilon, R_{\text {des }}=2, c_{1}=10$, $c_{2}=1.05, \alpha=0.02, T(n)=\frac{1}{0.01 \ln n}$, and $\tau=20$. The desired configuration (global minimizer of $U$ ) is shown in Fig. 3 (b) (modulo vehicle permutation and formation translation on the grid). Simulated annealing was performed for $10^{4}$ steps.

The sensor error was modeled as additive noise $Z_{x}$, as in (15). A uniform distribution was selected for $Z_{x}$. Other distributions can be studied accordingly. The potential difference of the example was calculated to be $\Delta_{U}=11$. So the potential error bound $\Delta_{z}$ should be less than 5.5 in order to guarantee convergence. In the simulations, we compared three different cases: noise-free, $\Delta_{z}=5$, and $\Delta_{z}=30$. Moreover, for comparison, we studied cases where the sensor error is modeled as additive white Gaussian noise (AWGN). Due to the lack of analytical results, numerical studies are provided instead. Two different variances, $\sigma=1$ and 5 , were used in the simulations respectively.

To demonstrate the trend of convergence to the lowest potential, one can calculate the error $\left\|\nu_{n}-\Pi_{\infty}\right\|_{1}$ as metric, where $\nu_{n}$ is the empirical distribution of configurations (again modulo vehicle permutation and network translation), and

$$
\Pi_{\infty}(x)=\left\{\begin{array}{l}
1 \text { if } x \text { is desired } \\
0 \text { otherwise }
\end{array}\right.
$$

Therefore,

$\left\|\nu_{n}-\Pi_{\infty}\right\|_{1}=1-\nu_{n}\left(x^{*}\right)+\mid 0-\left(1-\nu_{n}\left(x^{*}\right) \mid=2\left(1-\nu_{n}\left(x^{*}\right)\right)\right.$,

where $x^{*}$ denotes the desired formation. The evolution of $\left\|\nu_{n}-\Pi_{\infty}\right\|_{1}$ for different potential error bounds is shown in Fig. 4 , where $\nu_{n}\left(x^{*}\right)$ is calculated as the relative frequency of sampling $x^{*}$ in 1000 annealing steps. The plot suggests that when the potential error bound $\Delta_{z} \leq \frac{1}{2} \Delta_{U}$, the convergence trend is roughly the same as in the noise-free case. On the other hand, when $\Delta_{z}$ is relative large, the convergence trend is barely observed.

With the sensor random-error being modeled as AWGN, similar convergence properties were observed in simulations. For a normal distribution, 99.7\% samples lie in $[-3 \sigma,+3 \sigma]$, which is roughly comparable to the former cases with $\Delta_{z}=6 \sigma$. Hence, the case $\sigma=1$ should be comparable with the case $\Delta_{z}=5$, and the case $\sigma=5$ corresponds to the case $\Delta_{z}=30$. In the simulations, it was observed that the convergence rate with $\sigma=1$ is slightly faster than the case $\Delta_{z}=5$ with uniform distribution. Similar results were observed by comparing cases $\sigma=5$ and $\Delta_{z}=30$. The reason is the bell shape of the normal distribution, where probability densities concentrate at the center.
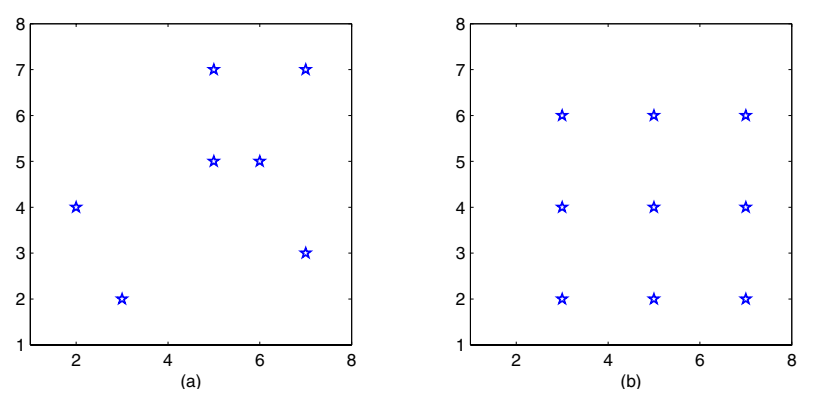

Fig. 3. The initial and desired configuration for 9 vehicles. (a) Initial configuration; (b) Desired configuration

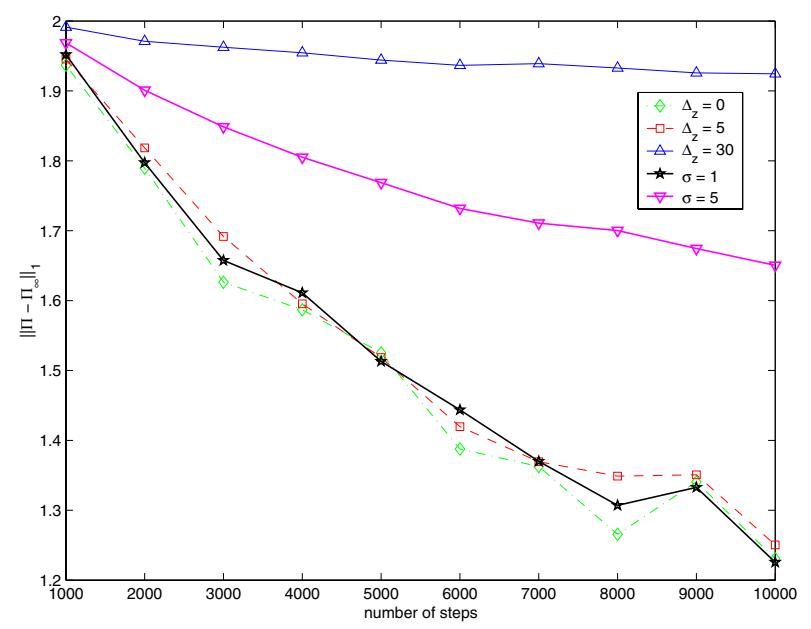

Fig. 4. Comparation of the evolution of $\left\|\nu_{n}-\Pi_{\infty}\right\|_{1}$ for different sensor noise. 


\section{Summary AND CONCLUSIONS}

In this paper, the impact of potential function inaccuracy on the convergence of a stochastic path planning algorithm, a simulated annealing algorithm based on Gibbs sampler, was investigated. Two types of potential error, range-error and random-error, were studied. It was shown that if the bound of the range-errors is less than half of $\Delta_{U}$, the annealing algorithm would yield the same limiting configuration(s) as original one(s).

By augmenting the state space, at a fixed temperature, the Gibbs sampling with random-errors was formulated as a Markov chain whose transition probability has the form $\tilde{p}_{T}(x, y)=\int p_{T}(x, y \mid Z=z) f(z) d z$. By further assuming that the random-errors have limited support, it was shown that the annealing algorithm yields desired configuration(s) if the error bound satisfies $\Delta_{z} \leq \frac{1}{2} \Delta_{U}$. The results were confirmed with simulations.

Furthermore, it is of interests to study cases where the random-error lives on unlimited support. For simplicity, AWGN was used to compare with the limited support cases. Interestingly, we found that AWGN has similar convergence properties as the limited support cases. Instead of using $\Delta_{z}$, simulations suggest that $6 \sigma$ might be a good indicator used for testing the convergence condition of proposition 3.5. For future work, it would also be interesting to extend our convergence analysis to random-errors with unlimited support.

\section{REFERENCES}

[1] J. S. Baras and X. Tan, "Control of autonomous swarms using Gibbs sampling," in Proceedings of the 43rd IEEE Conference on Decision and Control, Atlantis, Paradise Island, Bahamas, 2004, pp. 47524757.

[2] W. Xi, X. Tan, and J. S. Baras, "Gibbs sampler-based path planning for autonomous vehicles: Convergence analysis," in Proceedings of the 16th IFAC World Congress, Prague, Czech Republic, 2005.

[3] — , "A stochastic algorithm for self-organization of autonomous swarms," in Proceedings of the 44th IEEE Conference on Decision and Control, Seville, Seville, Spain, 2005.

[4] — - "A hybrid scheme for distributed control of autonomous swarms," in Proceedings of by the 24th American Control Conference, Portland, Oregon, 2005.

[5] —_ "Gibbs sampler-based coordination of autonomous swarms," Automatica, vol. 42, no. 7, 2006.

[6] D. A. Schoenwald, "AUVs: In space, air, water, and on the ground," IEEE Control Systems Magazine, vol. 20, no. 6, pp. 15-18, 2000.

[7] J. R. T. Lawton, R. W. Beard, and B. J. Young, "A decentralized approach to formation maneuvers," IEEE Transactions on Robotics and Automation, vol. 19, no. 6, pp. 933-941, 2003.

[8] A. Jadbabaie, J. Lin, and A. S. Morse, "Coordination of groups of mobile autonomous agents using nearest neighbor rules," IEEE Transactions on Automatic Control, vol. 48, no. 6, pp. 988-1001, 2003.

[9] H. G. Tanner, A. Jadbabaie, and G. J. Pappas, "Stable flocking of mobile agents, Part I: Fixed topology," in Proceedings of the 42nd IEEE Conference on Decision and Control, Maui, Hawaii, 2003, pp. 2010-2015.

[10] R. Olfati-Saber and R. M. Murray, "Consensus problems in networks of agents with switching topology and time-delays," IEEE Transactions on Automatic Control, vol. 49, no. 9, pp. 1520-1533, 2004.

[11] R. Olfati-Saber, "Flocking for multi-agent dynamic system: Algorithms and theory," IEEE Transactions on Automatic Control, vol. 51, no. 3, pp. 401-420, 2006.
[12] N. E. Leonard and E. Fiorelli, "Virtual leaders, artificial potentials and coordinated control of groups," in Proceedings of the 40th IEEE Conference on Decision and Control, Orlando, FL, 2001, pp. 29682973.

[13] P. Song and V. Kumar, "A potential field based approach to multirobot manipulation," in Proceedings of the IEEE International Conference on Robots and Automation, Washington, DC, 2002, pp. 12171222.

[14] P. Ogren, E. Fiorelli, and N. E. Leonard, "Cooperative control of mobile sensor networks: Adaptive gradient climbing in a distributed environment," IEEE Transactions on Automatic Control, vol. 49, no. 8, pp. 1292-1302, 2004.

[15] D. H. Kim, H. O. Wang, G. Ye, and S. Shin, "Decentralized control of autonomous swarm systems using artificial potential functions: Analytical design guidelines," in Proceedings of the 43rd IEEE Conference on Decision and Control, vol. 1, Atlantis, Paradise Island, Bahamas, 2004, pp. 159-164.

[16] Y. Koren and J. Borenstein, "Potential field methods and their inherent limitations for mobile robot navigation," in Proceedings of the IEEE International Conference on Robotics and Automation, Sacramento, CA, 1991, pp. 1398-1404.

[17] R. Volpe and P. Khosla, "Manipulator control with superquadric artificial potential functions: Theory and experiments," IEEE Transactions on Systems, Man, and Cybernetics, vol. 20, no. 6, pp. 14231436,1990

[18] J. Kim and P. Khosla, "Real-time obstacle avoidance using harmonic potential functions," IEEE Transactions on Robotics and Automation, vol. 8, no. 3, pp. 338-349, 1992.

[19] J. Barraquand, B. Langlois, and J.-C. Latombe, "Numerical potential field techniques for robot path planning," IEEE Transactions on Systems, Man, and Cybernetics, vol. 22, no. 2, pp. 224-241, 1992.

[20] C. Liu, M. H. A. Jr, H. Krishna, and L. S. Yong, "Virtual obstacle concept for local-minimum-recovery in potential-field based navigation," in Proceedings of the IEEE International Conference on Robotics and Automation, San Francisco, CA, 2000, pp. 983-988.

[21] X. Zou and J. Zhu, "Virtual local target method for avoiding local minimum in potential field based robot navigation," Journal of Zhejiang University Science, vol. 4, no. 3, pp. 264-269, 2003.

[22] G. Winkler, Image Analysis, Random Fields, and Dynamic Monte Carlo Methods : A Mathematical Introduction. New York: SpringerVerlag, 1995.

[23] L. K. Grover, "Simulated annealing using approximate calculation," in Progress in Computer Aided VLSI Design, 1989.

[24] S. B. Gelfand and S. K. Mitter, "Simulated annealing with noisy or imprecise energy measurements," Journal of Optimization Theory and Applications, vol. 61, no. 1, pp. 49-62, 1989.

[25] _ "Simulated annealing with noisy or imprecise energy measurements," Algorithmica, vol. 6, no. 3, pp. 419-436, 1991.

[26] D. R. Greening, "Simulated annealing with errors," Ph.D. dissertation, UNIVERSITY OF CALIFORNIA Los Angeles, 1995.

[27] P. Bremaud, Markov Chains, Gibbs Fields, Monte Carlo Simulation and Queues. New York: Springer Verlag, 1999.

[28] S. Geman and D. Geman, "Stochastic relaxation, Gibbs distributions and automation," IEEE Transactions on Pattern Analysis and Machine Intelligence, vol. 6, pp. 721-741, 1984. 